\title{
Influence of Male Presence on the Social Structure of Lesser Spotted Dogfish (Scyliorhinus canicula) Female Groups
}

\section{Micarelli $\mathrm{P}^{1 *}$, Pieraccini $\mathrm{F}^{1}$, Reinero $\mathrm{FR}^{1,2}$ and Sperone $\mathrm{E}^{2}$}

${ }^{1}$ Sharks Studies Centre-Scientific Institute, Italy

${ }^{2}$ Department of Natural Science, University of Calabria, Italy

*Corresponding author: Micarelli Primo, Sharks Studies Centre, Loc.Valpiana, Massa Marittima, GR, Italy, Tel: 00393896732796; Email: primo.micarelli@gmail.com; direzione@ centrostudisquali.org

\section{Research Article \\ Volume 4 Issue 1}

Received Date: January 06, 2020

Published Date: January 26, 2020

DOI: $10.23880 /$ ijoac- 16000179

\section{Abstract}

Sexual segregation in the lesser spotted dogfish (Scyliorhinus canicula) is most likely driven by the female strategy of avoidance of the males, which causes the formation of female groups in the nooks and shelters on the seafloor. This strategy allows them to reduce the multiple events of male sexual advances, which are energetically expensive. In order to examine the existence of a social structure in female lesser spotted dogfish aggregations and the impact of the presence of males on them, we analyzed the structure of the social network and the temporal stability of the associations of two populations each one including five females. An easy insertion of the males in female population was made possible by the presence of a homogeneous initial social structure with quite weak bonds. The social structure is regulated by differences in the individual behaviour, resulting in different strength and stability of aggregations of conspecifics.

The addition of the males produced a reduction of the Average Association Index and of the measures related to the populations' network, strength of the associations and level of indirect connections of the individual. The presence of the males seems to have a greater effect on the individuals that are more weakly linked; while key individuals typically reduce their network values, but remain well integrated in the population; peripheral individuals have more variables values. This result indicates that relations exist between the sex, which is in contrast with the hypothesis of the female avoidance of males, which should produce strong and stable female groups. The actual knowledge of the behaviour and distribution of the Mediterranean lesser spotted dogfish does not prove the existence of a sexual segregation in this species. Based on the results we can assume that the home-ranges of females and males are not separated and that the male presence does not trigger a female aggregation mechanism. It is noteworthy that males have a destructive effect on the female associations, as these ones have shown a reduction of all the values. This might suggest that, in nature, the male presence and therefore the potential male sexual coercion may cause the spatial dispersion of the individuals of the population and reduce the cohesion of the group.

Keywords: Scyliorhinus canicula; Social Networks; Home Range

\section{Introduction}

\section{Sex Differences in Behaviour of Scyliorhinus canicula}

The lesser spotted dogfish, Scyliorhinus canicula, is a marine vertebrate exhibiting sexual segregation. This species is sexually monomorphic in body size. Its relatively high abundance in the Mediterranean Sea and its wide coastal distribution, together with its relatively small adult size, makes the lesser spotted dogfish a good model to investigate the causes that lead to differences in sexual behaviour and 
sexual segregation. However, while for this species there is a great deal of information on the physiology $[1,2]$, diet and reproductive cycle, little is known about its behaviour in nature [3,4]. Rodriguez-Cabello, et al. [5] has shown that this species does not travel long distances. Sims, et al. [6] found that males and females of S. canicula exhibit alternative behavioural strategies. In the south of Ireland (Lough Hyne, brackish lake), they observed that males are active during dusk and night and moved from a depth of $12-24 \mathrm{~m}$ to $3 \mathrm{~m}$ for feeding. On the contrary, females take refuge in shallow water ( 0.5 to $1.5 \mathrm{~m}$ ) in ravines and caves during the day, and moved to deeper water only once every two to three nights. Hence, the home-ranges of the two sexes appear spatially separated [6]. More recently, Sims, et al. [7] demonstrated the existence of daily vertical migrations in the species living closer to the seabed. By using short- and long-range acoustic telemetry, they showed that during daytime the males of lesser spotted dogfish undertake movements along the bottom or along the rocky slopes, where temperatures are cooler, while during night-time they are heading for shallowest areas, warmer and prey-rich. The results suggested that males forage in warm water and prefer to rest and digest in colder water. The energy model of this strategy "warm hunt-cold rest" indicated a reduction in the daily energy costs of about $4 \%$, meaning that males of S. canicula used these daily vertical migrations as an energy conservation strategy to increase metabolic bioenergetics-efficiency [7]. Actually, sex segregation is a general feature of the shark populations, though this is yet to be investigated in most species. In a review of sex differences in habitat selection and reproductive strategies of sharks Sims [8] used S. canicula as a model species, through which it is possible to test the hypotheses on sexual segregation. The hypothesis of food selection was discarded, because the two sexes do not show differences in the niche food. The assumption of the risk of predation was hardly likely because the lesser spotted dogfish are subject to low levels of season predation at the study site. It is thought that the sexual segregation of S. canicula is due to the fertility-thermal niche hypothesis and to social factors. Females typically choose areas with warmer habitat than males [9]. In the brackish lake of Lough Hyne, the use of the females to take refuge in shallow water during daytime exposes them to temperatures exceeding $18^{\circ} \mathrm{C}$ in August and September, while the males during day time stay in deeper waters, exposed to temperatures not higher than $15.7^{\circ} \mathrm{C}$ [10]. Even if a relation has been suggested between the selection of warmer habitat and increased rates of embryonic development in females, there is still no direct evidence. In contrast, the male of the lesser spotted dogfish, actively chooses colder water, in spite of reducing the opportunities for foraging in the short term [7]. However, the choice of colder waters by the males can be linked at the optimum temperature for sperm production [11]. Mating in elasmobranchs involves a long-lasting series of complex behaviours. A female can be pursued by several males and can present lesions due to their aggressiveness. During mating, the S. canicula female is involved in an extreme twist by the male, which rolls tightly around the pelvic region of the female [2]. The mating can be lengthened not only because of the males competing for a single female, but also because the mating itself requires maintaining the body contact and the posture control. However the females of S. canicula (as many other species of elasmobranchs) are known to store sperm, suggesting that the mating does not necessarily have to precede ovulation in this species. To maintain their fertility and the possibility of growth, the females of lesser spotted dogfish may therefore seek to limit the mating activity, which is energetically very expensive. Therefore, the existence of a unisexual refuge could help to reduce the levels of sexual harassment of male to females and can facilitate female choice. Nevertheless, while some assumptions for sexual segregation have been partially examined in this species, further investigations are necessary to fully test these hypotheses [12].

\section{Social Structure}

Biological social structures are often explained in terms of networks of direct and indirect interactions [13]. These biological networks are often highly dynamic and may be subject to a variety of biotic disturbances, from intraspecific competition for food to intersexual harassment, and this may lead to behaviours promoting a high variety of the individuals, each one trying to optimize his own fitness. Hence, the behaviour of each individual group member can potentially affect the overall dynamic of a social network [14]. Experimental research into the social behaviour and group dynamics of fish has been almost exclusively limited to freshwater teleosts $[15,16]$, because such models usually display short life histories/generation times, high numbers of offspring, and small size at maturity. The Elasmobranchs are not a good model because they show k-selected features, in that they are long lived and slow to gain sexual maturity, they frequently reach adult lengths of more than $1 \mathrm{~m}$, and consequently they often occupy apex positions within their ecological niche.

This is the first study that tries to interpret the direct effects of the male presence on groups of females of Mediterranean Scyliorhinus canicula.

In this study the main objective was to assess how much and how far the male presence may influence the social grouping behaviour of females, with the aim of evaluate the structure of female social networks of lesser spotted dogfish and also the influence of the male presenceon:

- The dynamics of female networks based on temporal stability analyses.

- The activity of females with different degrees of social 
connectivity.

Data obtained from catches of Mediterranean lesser spotted dogfish indicate that sexual segregation is much less marked in Mediterranean populations, and it is also possible that the male presence does not alter at all the social structure of the females [17].

\section{Materials and Methods}

\section{Data Collection}

In November 2013 ten mature females of S. canicula were caught off the Elba Island by the fishing vessel "Mare Blu", by using trawls with a mesh size of $40 \mathrm{~mm}$. Eight mature males, which are also object of this study, were caught in the same manner during the same period. After the capture, sharks were brought to the Sharks Study Center-Scientific Institute, located in Valpiana (Massa Marittima, Gr). The sharks were then placed in special tanks and held in quarantine. The mature females chosen for the experiment were measured and weighed. Length was used as an indicator of the maturity status of the individuals and weight was needed to assess the quantity of food to be provided during the study period. Measurements of length and weight were repeated at the end of the experiment to ensure that captivity did not affect the animal welfare. Mature females having a similar length were chosen, with the smallest measuring $39 \mathrm{~cm}$ and the largest $43.5 \mathrm{~cm}$, in order to avoid any possibility of segregation according to body size. Finally, all the sharks were marked with differently colored polyester threads, permitting to distinguish the individuals during each observation. The behaviour of ten other specimens of S. canicula marked with the same technique had been observed in the months before the experiment, in order to check that marking did not affect their behaviour. The tags were applied to the experimental animals seven days before starting the data collection, a time considered necessary and sufficient for the recovery of the stress accumulated during the operation. Colored tags could easily be used since $S$. canicula is not able to see and distinguish colors, as its eyes are adapted to scotopic view
[18]; this avoid that the presence of colored tags can play some role in the choice of partners. Initially two populations of five females were formed, and each one was placed in its own experimental tank. Both tanks had a size of 201 x 100 $x 31 \mathrm{~cm}$, a resting area surface of $2.0 \mathrm{~m} 2$ and a capacity of $400 \mathrm{~L}$ of sea water. The experimental arenas and their populations were defined as T3 and T4. The water in each tank belonged to a closed system filtered by two mechanical/ biological horizontal filters. Temperature, salinity, $\mathrm{pH}$ and nitrite were checked regularly in each tank to ensure that none of these parameters would alter behavior, neither influence the well-being of the animals. Data collection on the partner preferences and the association models was performed through a series of visual scans having a period of eight hours per day, five days per week between 10:00 a.m. and 06:00 p.m. The data were obtained through 13 scans per day per arena, every $40 \mathrm{~min}$. During each visual scanning, the location of the animals was recorded in terms of proximity to other individuals. The strength of the association was calculated based on the frequency of samplings in which individuals were observed to rest together. The considered behaviors are shown in Table 1. In this study we evaluated patterns of association between individuals according to a spatial proximity measure. Although relational data based on associations offer less information than those based on interactions, they were considered to be the most useful for this study because in this species individuals spend most of the daytime resting and have very few interactions (events). In this work, to define a group, we used the sense of touch for identifying the strongest relationships, while we defined the weakest one by the method of "Gambit of the group". The method used is the standard one, considering the group membership of an individual on the basis of the distance of the front half of the body with respect to a neighbor. The considered portion of the body is the head, defined as the region going from the tip of the rostrum to the last gill slit [19]. Only symmetric associations were considered for this study, while the asymmetric ones were not considered important because this type of data may be difficult to interpret and to calculate using the SOCPROG 2.4 program.

\begin{tabular}{|c|c|c|}
\hline Behaviour & Description & Score \\
\hline Solitary resting & Resting > a head length from another individual. & 1 \\
\hline Solitary swimming & Swimming > a head length away from another individual. & 2 \\
\hline Resting together (Asymmetric) & Resting $\leq$ a head length from another individual. & 3 \\
\hline Tactil resting (Symmetric) & $\begin{array}{c}\text { Resting with the part of fusiform body touching another individual or } \\
\text { some pectoral bending. }\end{array}$ & 4 \\
\hline
\end{tabular}

Table 1: Definition of the visual behaviour recorded during each scan and relative associated scores, permitting to quantify the behavioral repertoires.

Both populations (T3 and T4) were studied from 2

December 2013 to 23 February 2014. In the first four weeks 
of the study we investigated the population social structure by evaluating the behaviour of five females per every tank. This first observation period allowed us to define the initial social structure by identifying the specimens which were more or less linked. Then we inserted two males in each arena for two weeks. After a week, enabling the females to recover from the stress, we replaced the two males of each arena with two other specimens, for two more weeks. Finally, we removed the last couple of males from the tanks and then we checked again the social structure of the populations formed by the females. The couples of males to be introduced in the tanks were introduced $24 \mathrm{~h}$ before starting the observations, in order to allow the animals to acclimate within the experimental arena. All the males used in the experiment were different specimens, having about the same length of the experimental females. The introduced males were not marked, as they could be easily recognized for their different colors and the different arrangement of the spots in the back-cephalic area. The animal husbandry and experimentation protocols were reviewed and approved in accordance 166 with the Directive 2010/63/EU.

\section{Statistical and Data Analyses}

Data were analysed with the SOCPROG 2.4 software [20]. In this study SOCPROG 2.4 was used to define the social structure of lesser spotted dogfish females held in captivity based on the symmetric association behavior "Tactile rest." The program allowed creating arrays of the association indices, which were used for the permutation test, the network analysis and the temporal analysis. The first operation was to choose which index of association to use; this is a fundamental building block upon which the study and description of the social structure is made. Almost all the association indices estimate the proportion of time that a pair of individuals spends in association, so that such indices are symmetrical (the index of association of $A$ with $B$ is equal to that of $B$ with $A$ ) and vary between 0 (no association) and 1 (very strong association). Since the individuals are in a controlled environment and it is always possible to observe and identify the animals, no inferences are needed on the time when the animals are not observed.

Hence, captivity provides a very stable situation, allowing collecting an ideal data set underlying these assumptions:

- The registered association is a symmetric measure 1: 0 , based on the fact that members of a dyad are or are not associated during the sampling period.

- The registered associations are very accurate.

- If an individual is identified during a sampling period, then his associates are also identified.

- The members of a dyad have equal probability of being identified whether they are members or not.
As these assumptions are met, then the best index that can be selected is the "Simple Ratio Index" which simply corresponds to the ratio between the number of sampling periods in which the two individuals are registered as associated and the number of sampling periods in which it at least one of the two is identified. In this situation, this index is the most objective estimation of the duration of the time that the couple spends together [21].

The Simple Ratio Index is calculated as follows:

$$
S R I=X /(X+Y a b+Y a+Y b)
$$

where $X$ is the number of observations in which the individuals $a$ and $b$ are observed when resting together in the same group, whilst $Y a b$ corresponds to the number of sampling periods in which $b$ is observed in other groups than $a, Y a$ is the number of sampling periods in which only $a$ is observed and $Y b$ is the number of sampling periods in which only $b$ is observed [21]. Symmetrical associations related to "Tactile rest" have been obtained based on contacts that have taken place between the animals during the rest behaviour.

\section{Permutation Tests for the Preferred / Avoided Association}

We used the randomisation procedure described by Whitehead [20] to determine if there were significant differences between the patterns of association observed within the two populations before, during and after social manipulations. Data were obtained from observations to test the null hypothesis of individuals being associated by chance, against the alternative hypothesis of animals having preferences in the group. We used Monte Carlo permutation tests, based on the procedures outlined by Bejder, et al. [22]. The permutation method chosen is "Exchanges all groups" as recommended in studies in which all animals are likely to be seen in the same study area and the groups are sampled independently. In the calculation procedure 40000 permutations were made with 1000 flip of the data set permutation [20], in order to stabilize the P-value. This procedure was used to isolate the pairs of individuals associating or avoiding each other more than expected under random conditions.

\section{Network Analysis}

The Network Analysis configures a social system as a web, consisting of a set of nodes (points), which correspond for example to individuals, connected by means of edges (lines) indicating interactions. The networks are similar to sociograms and dendrograms, as they are graphical representations in which relationships are indicated by means of links. The Sociogram is a representation of a 
measure of relationship in which individuals are represented as points (nodes) in a plane, and the lines that show the links (edges) are drawn to each other, indicating the strength of the relationship. SOCPROG 2.4 uses arrays of association indices as a weighted network (not 1: 0) to produce the values of "Average Path Length or Reach", "Eigenvector Centrality" and "Weighted Degree or Strength".

- Average Path Length or Reach (R.): Reach is a measure that indicates the indirect connection of an individual within the population; for a binary network it has been defined as the smallest number of nodes between 2 individuals, and describes the extent to which each member of a network can reach the other members of that network $[23,24]$.

- Eigenvector Centrality (E.C.): Eigenvector Centrality is a measure of how well an individual is connected within the network [25]. For each individual, this value gives us a number indicating its connectivity within the network.

- Degree or Weighted Strength (S.): The strength is simply the sum of all the association indices of an individual with the others. For a weighted network of association indices, the Strength is the ability of an individual to be sociable.

It is necessary to emphasize that these measures were derived exclusively from the symmetrical behaviour of "Tactile rest" and therefore they were weighed in the association indexes. Such measures do not only provide some indications about the strength of the social structure, but also allow assessing the relative importance to be attributed to an individual, and his influence on the social network [26]. The Network Analysis is basically used for two reasons: it provides formal descriptors characterizing social groups and quantitative measures of the relations, allowing testing models on relationships and social structure [27]. The Social Network Analysis should explain biological phenomena much better than other measures such as the "group size", which does not show details about individual social relationships.

\section{Temporal Stability}

The temporal pattern of relationships is one of the main elements, nevertheless it is often mistreated in the conceptual work on social organizations [28]. By considering how associations, interactions and relationships change over time, the understanding of social life of an animal and its society turns out to be a key aspect. The main method that SOCPROG 2.4 uses to examine the temporal pattern of social relations is the LAR, "Lagged Association Rate" [29]. The Lagged Association Rate of population corresponds to the probability of the occurrence of an association, $\pi$ units of time after a previous association averaged over all associations [30]. Essentially the Lagged Association Rate is an estimate of the probability that individuals being associated in a given time will also be associated $\pi$ units of time later [31]. For considering the Lagged Association Rate in perspective, the calculation of the NAR (Null Association Rate) value helps us. This is the expected value of the Lagged Association Rate when no preferred associations are found between the pairs, given the number of associations of each individual in each sampling period [20]. When there are not preferred associations, the probability of being associated of two individuals, $\mathrm{A}$ and $\mathrm{B}$, is independent from the fact that they have been previously associated. The Null Association Rate is generally less than or equal to the Lagged Association Rate. When the Lagged Association Rate is equal to the Null Association Rate on a series of time intervals, then there isn't a preferred association between two individuals during this time period. In SOCPROG, the LARs (and NARs) are represented as a function of the time intervals, using the "moving average" method [20]. The Lagged Association Rate and its associated time interval are calculated on a 400 associations for all analyses. The temporal dynamics of these networks is shown by the information "Quasi-Akaike smaller Criterion" (QAIC) [32]. Unless otherwise indicated, there is substantial support for all models based on a $\triangle$ QAIC value between 0 and 2 [20]. In order to obtain a precise evaluation of the Lagged Association Rates and the parameters of fitted models, SOCPROG uses the Jacknife procedure, in which the analysis is run several times omitting each time a different sampling period or set of sampling periods [32].

\section{Results}

\section{Initial Period of Social Definition}

With data restricted to symmetric (or tactile) association, the permutation test showed a strong difference from random association in both groups for the 4-week period in which the initial social structure was analysed. The test for preferred or avoided association showed that during the initial period of social definition there was a significant difference between the observed mean association and the randomized mean association (Table 2). Interestingly, the randomized mean association was greater than the observed one, suggesting that the avoidance behavior was more prevalent than the association one. This also suggests that preference and avoidance behaviors between individuals occurred significantly more often than expected under the null hypothesis of a random association.

The observation of behavioral states during the initial period of the experiment allowed to determine the presence or absence of a social structure within the two populations of females of lesser spotted dogfish. In addition to this assessment, it was also possible to identify the key female 
of the structure (the best integrated in the structure) and the peripheral female (the loneliest one). The T3 and T4 populations had a homogeneous social structure, as individuals did not exhibit great variability in the strength of the bonds, which are equally distributed (T3: Reach range =
1,02-1,52; Weighted Strength range $=0,84-1,20$; Eigenvector centrality range $=0,33-0,51$. T4: Reach range $=1,05-1,51$; Weighted Strength range $=0,79-1,48$; Eigenvector centrality range $=0,32-0,53$ ).

\begin{tabular}{|c|c|c|c|c|}
\hline \multirow{2}{*}{ Data set } & \multicolumn{3}{|c|}{ Mean Association Index } & \multirow{2}{*}{ P value (<0,001) } \\
\cline { 2 - 4 } & Tank & Observed & Randomized & \\
\hline \multirow{2}{*}{ Initial period } & T3 & 0,28686 & 0,39321 & 0,00000 \\
\cline { 2 - 5 } & T4 & 0,29046 & 0,40966 & 0,00000 \\
\hline \multirow{2}{*}{ First male introduction } & T3 & 0,19133 & 0,24772 & 0,00003 \\
\cline { 2 - 5 } & T4 & 0,24265 & 0,36936 & 0,00003 \\
\hline \multirow{2}{*}{ Second male introduction } & T3 & 0,23265 & 0,39519 & 0,00000 \\
\cline { 2 - 5 } & T4 & 0,19812 & 0,29845 & 0,00003 \\
\hline \multirow{2}{*}{ Final recovery } & T3 & 0,23443 & 0,33491 & 0,00003 \\
\cline { 2 - 5 } & T4 & 0,19754 & 0,27203 & 0,00000 \\
\hline
\end{tabular}

Table 2: Permutation test for preferred or avoided association before and after manipulating the social structure.

\section{First Introduction of the Male Couple}

The introduction of the first pair of male led to a number of changes in the social structure of both populations (Figures $1 \& 2)$.

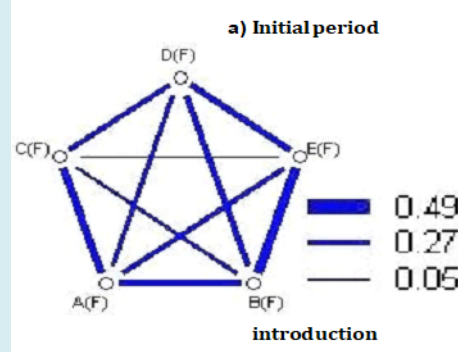

b) First males introduction introduction*
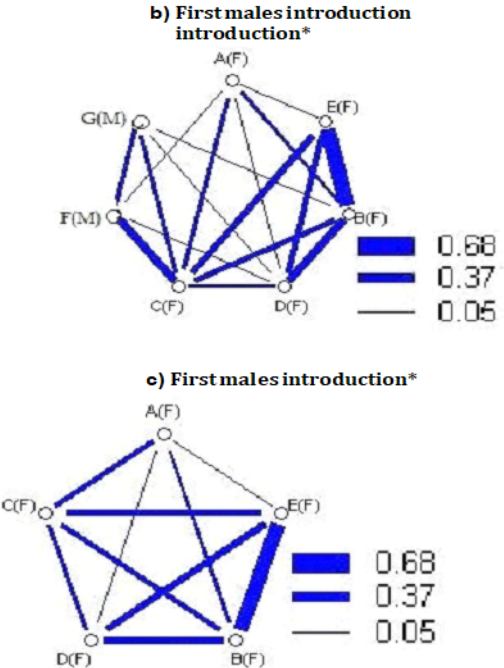

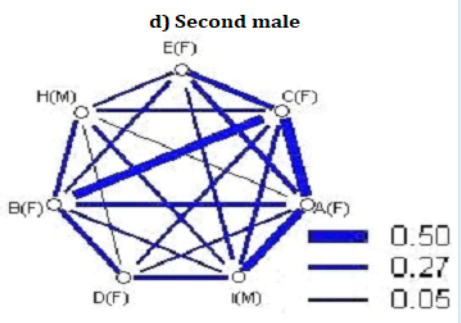

e) Second males
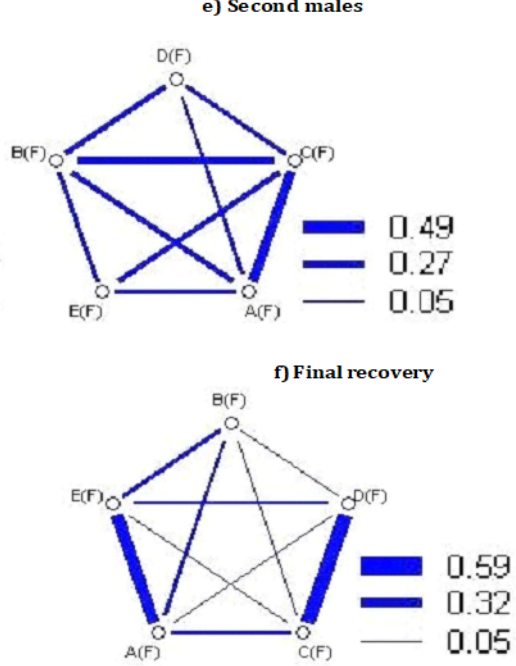

Figure 1: Symmetric sociogram (with relative tie weighting) of T3, showing the tactile resting behaviour of female lesser spotted dogfish. ${ }^{*}$ Sociograms with social structure of females exclusively during manipulation periods. 


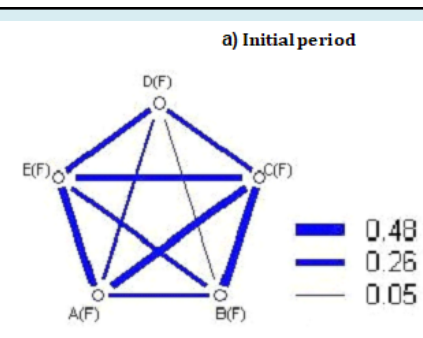

b) First males introduction

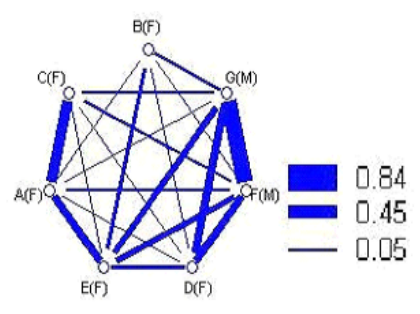

c) First males introduction*

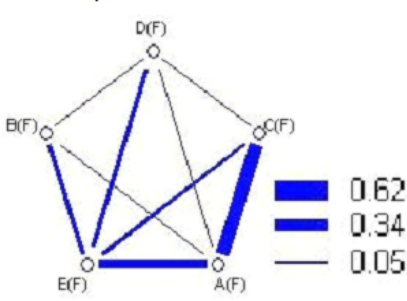

d) Second males introduction

A(F)

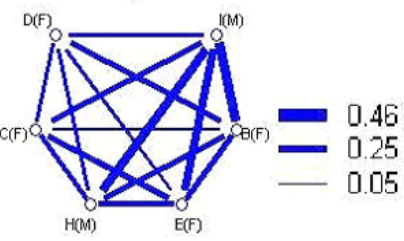

e) Second males introduction*

$A(F)$
0

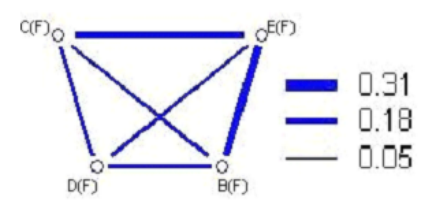

f) Final recovery

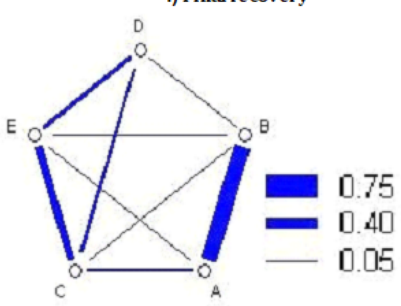

Figure 2: Symmetric sociogram (with relative tie weighting) of T4, showing the tactile resting behavior of female lesser spotted dogfish. * Sociograms with social structure of females exclusively during manipulation periods.

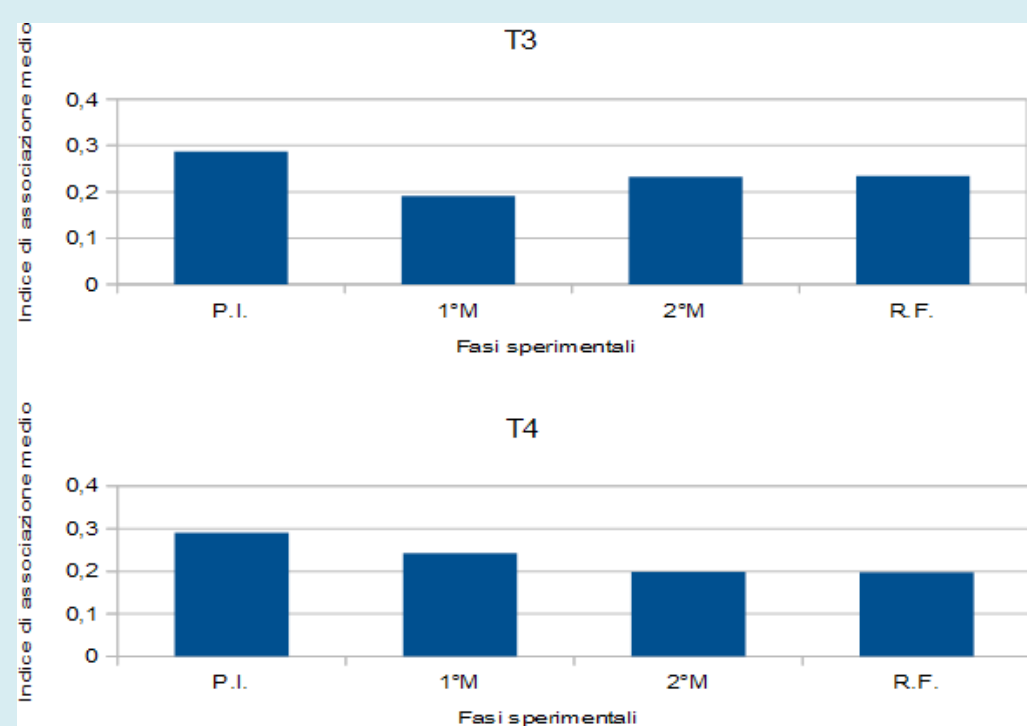

Figure 3: Mean Association Index across T3 and T4, for the initial social structure and manipulation periods. P.I.= initial period of social definition. $1^{\circ} \mathrm{M}=$ first male introduction. $2^{\circ} \mathrm{M}=$ second male introduction. R.F.= final recovery.

It is possible to see how they changed relations between females and males within the social networks of the females.
From Figure 3, showing the trends of the mean association Index of both populations during the various experimental 
stages, a reduction of the index is observed during this first period of social manipulation. The Strength of social ties remained the same in T3 (from 1,15 to 1,15) and it increased in $\mathrm{T} 4$ (from 1,16 to 1,46), suggesting more numerous associations between female and male sharks in this group. The other measures of the Network analyses showed equal values in T3 (Eigenvector centrality, from 0,44 to 0,43; Reach, from 1,34 to 1,36) and lower values in T4 (Eigenvector centrality from 0,44 to 0,42 ; Reach from 1,40 to 0,73 ). Not surprisingly, the sociograms (Figures $1 \& 2$ ) showed a fairly general reduction of the thickness of the lines connecting the various individuals compared to the sociograms of the social definition period; only in a few cases and in a few couples there is an increase of the thickness of the links.

\section{Second Introduction of a New Pair of Males and Final Recovery}

Also the second introduction of the new pair of males produced lowest levels of the mean association Index compared to those of the social definition period, confirming the destructive effect of the relations as in the previous phase of manipulation. Also in this period a distinct difference was observed in the Reach from the analyses with and without the data relating to the males, indicating that these took numerous contacts with the females (T3: Reach with males data $=2,04$; Reach without males data $=1,11$. T4: Reach with males data $=1,69$; Reach without males data $=0,36$ ). Also in this case the peripheral females had more contacts with males than key females, always according to the Reach values from the two analyses.

The period of final recovery, without any male, presented values of mean association Index, Strength, Reach and Eigenvector centrality that were quite consistent with the values obtained in the previous experimental stages. This may suggest that the destructive effect of males on female associations can be long lasting.

\section{Temporal Stability Analyses}

First period of social definition: Both $\mathrm{T} 3$ and $\mathrm{T} 4$ homogeneous populations showed a LAR with a downward trend. In one case (T4) it intersected the NAR, indicating a loss of temporal stability before the end of the phase of social definition, meaning that the individuals of this population showed preferred associations for shorter periods. The bestfitting model for all the arenas consisted of 2 levels of casual acquaintances:

$$
\text { a3 X exp(-a1 X td) +a4 X exp(-a2 X td), }
$$

where td represents time lag and $a 1, a 2, a 3$, and $a 4$ the exponential parameters of the model [20].

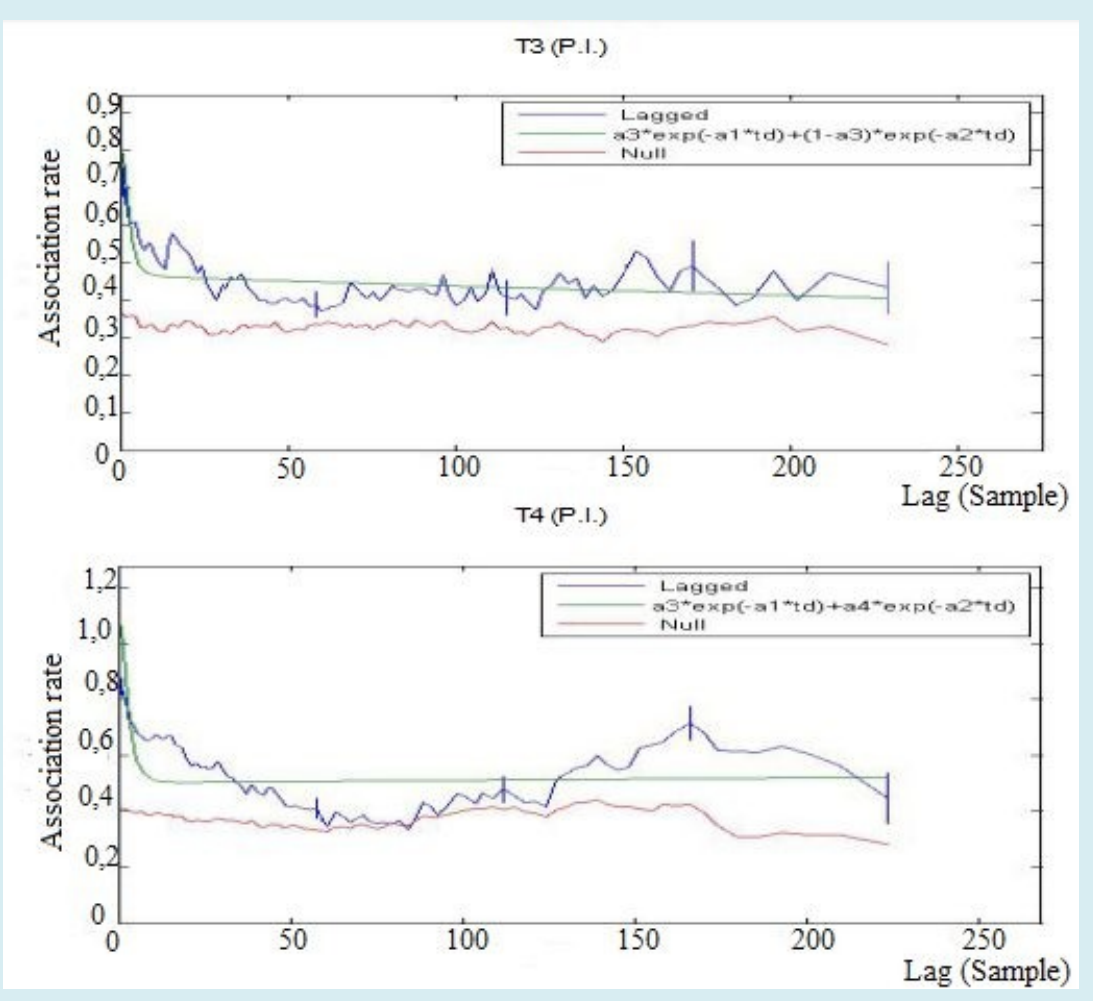

Figure 4: LAR (with jack-knifed estimates of precision) for initial social structure, in T3 and T4. LAR analyses were on symmetric associations and excluded any data from introduced individuals. 
From Figure 4 we see that the model chosen perfectly fits the T3 population but it presents a $\triangle \mathrm{QAIC}>2$ in the T4 population: for this reason another model was chosen for T4 with four exponential variables with two levels of casual acquaintances and a rapid disassociation.

First insertion of the male couple: In the T3 population, the LAR trend was descending and did not cut the NAR if not in the last time interval. This indicates that temporal stability was decreasing. The LAR of the T4 population followed a declining path towards the NAR, intersecting it in the second week of the experimental period, after about 80 time intervals. This indicates that in this population, which already in the social definition phase showed a temporal instability of relations, this tendency continued even with the presence of males. For all the populations the chosen model proved to be the best (Figure 5).
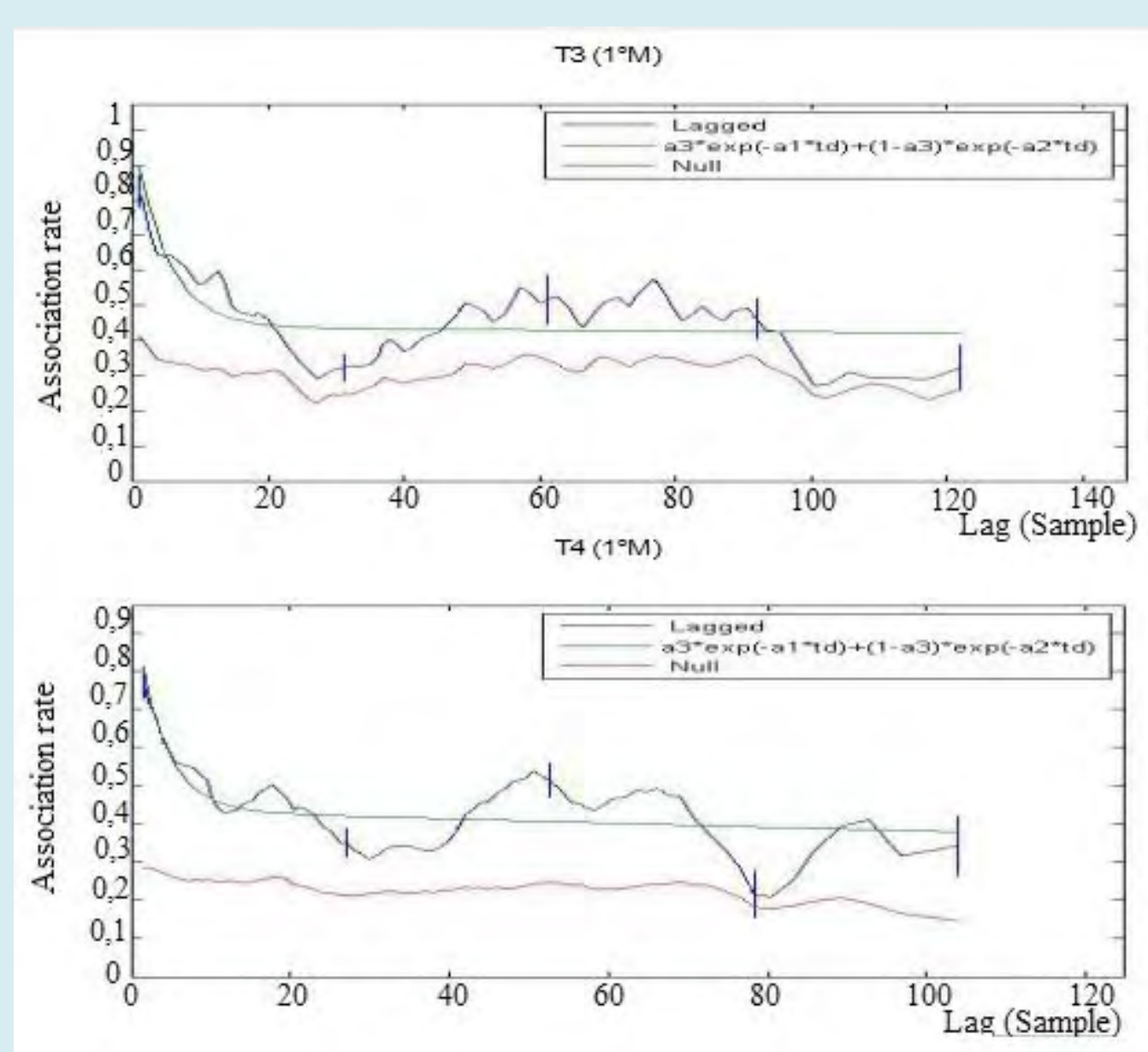

Figure 5: LAR (with jack-knifed estimates of precision) for first male introduction, in T3 and T4. LAR analyses were on symmetric associations and excluded any data from introduced individuals.

Second introduction of a new pair of males: In the second experimental phase, the LAR of population T3 showed a downward trend, but it never touched the NAR, although being very close. This indicates that the temporal stability lasted throughout the second period of experimental manipulation, with a tendency to lowering over the time. In the T4 population the introduction of the male determined a temporal instability already 3 days after their introduction. Therefore, females continue to show preferred associations only for rather short times. The chosen model showed to well fit all the populations (Figure 6). 


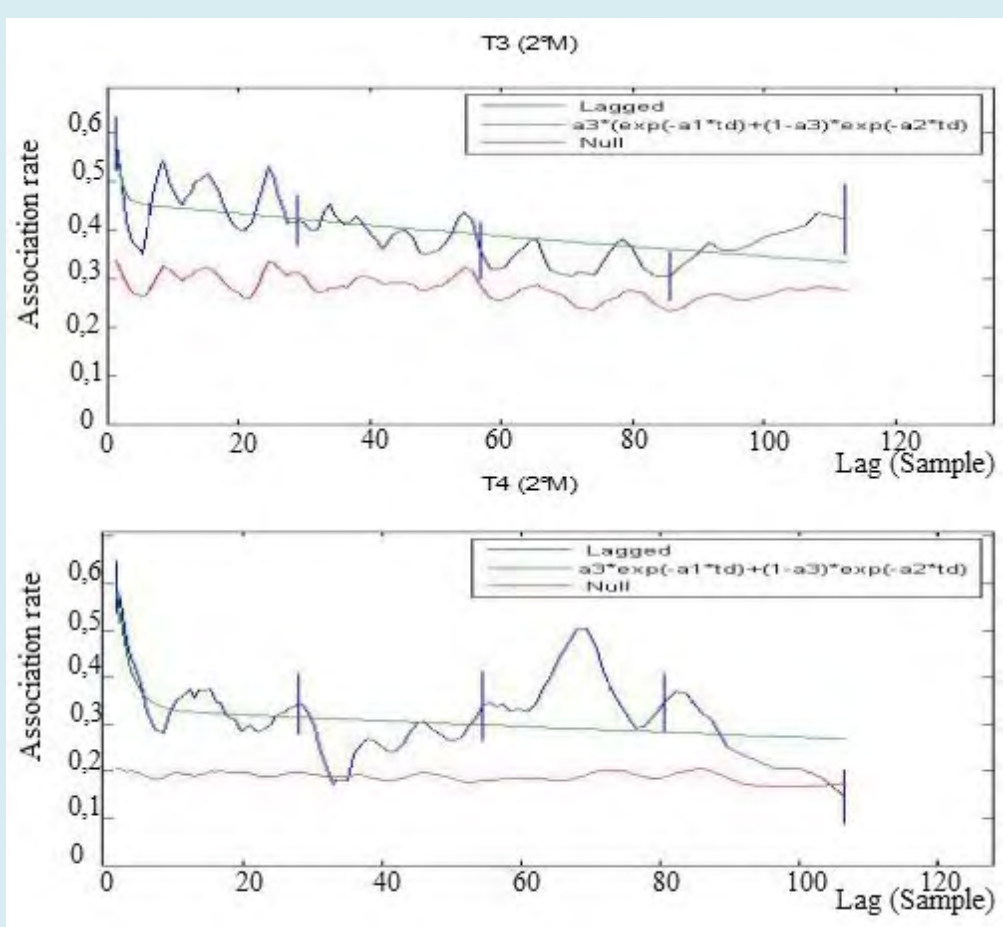

Figure 6: LAR (with jack-knifed estimates of precision) for second male introduction, in T3 and T4. LAR analyses were on symmetric associations and excluded any data from introduced individuals.

Final recovery: After a week of recovery from the stress

collection of association data.

suffered for the introduction of the males, we resumed the

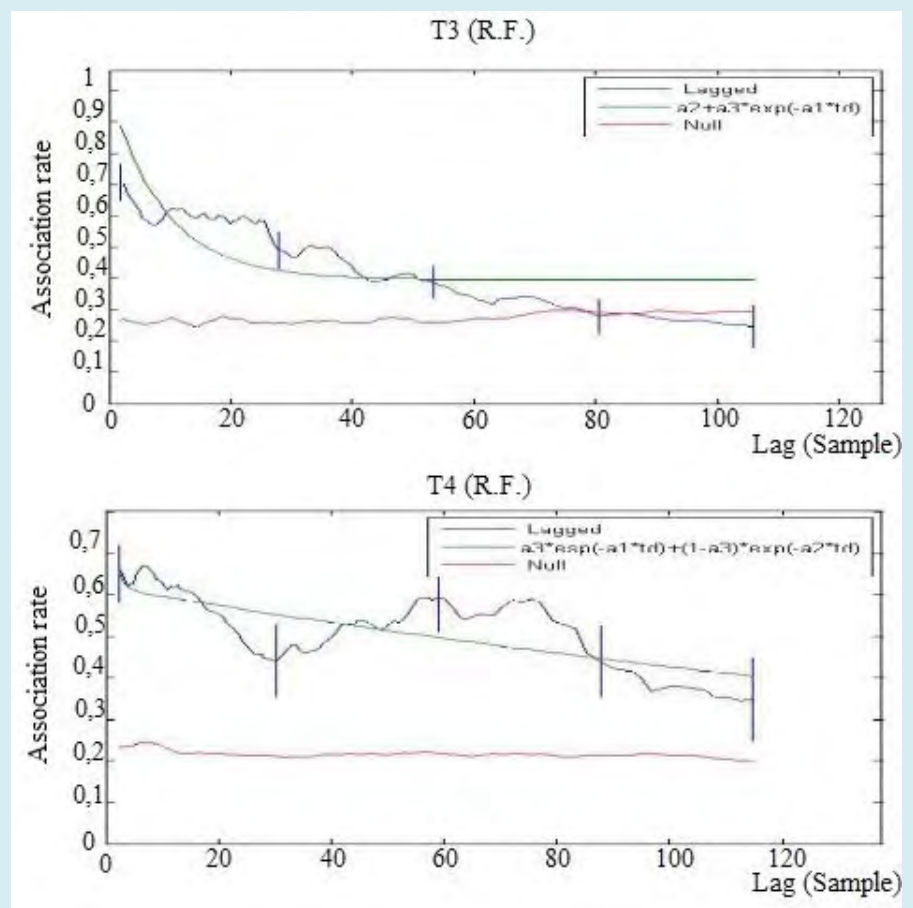

Figure 7: LAR (with jack-knifed estimates of precision) for final recovery, in T3 and T4. LAR analyses were on symmetric associations and excluded any data from introduced individuals. 
In T3 the LAR had a declining path and intersected the NAR after 80 time intervals, at the beginning of the second week, indicating that the preferred associations were maintained for quite short periods. We can suppose that males have a lasting disruptive effect on the female ties. Surprisingly, in T4 the LAR, although showing a declining path, did not cut the NAR and remained well above its level. The fit that better describing the trend of LAR in the T3 population had two variables and one level of casual acquaintances and one of rapid disassociation, because $\triangle \mathrm{QAIC}>2$; the model of $\mathrm{T} 4$ population is the chosen one (Figure 7).

\section{Discussion}

The asymmetrical parental investment of many animal species can strongly influence the sex ratio within a population [33]. The females of Scyluorhinus canicula adopt different behavioural strategies towards males and also show an overlap between the respective areas of activity $[6,7]$. This was partly attributed to an avoidance behaviour of the females towards the males, due to multiple forced copulations that are energetically expensive $[9,10]$. Since it has been observed that females of Scyliorhinus canicula aggregate into small but frequent groups within submarines shelters, often outside their thermal optimum [7], the effects of male coercion in this species can be significant. The females of lesser spotted dogfish can counteract the coercive behaviour by frequently changing their shelters with mass displacement, storing the sperm and delaying fertilization [8]. This is the first study that tries to interpret the direct effects of the masculine presence on females groups of Mediterranean Scyliorhinus canicula. A similar study was carried out by Jacoby [26] on Atlantic lesser spotted dogfish, confirming the results by Sims, et al. [6] showing an actual sexual segregation. In this study on Mediterranean lesser spotted dogfish, the first introduction of the males into the tanks with groups of females caused a reduction in the Index of average real partnership in all populations; the second insertion maintained fairly constant the real average association index, which was always smaller than during the social definition period. A decrease was observed also in the value of the overall average Strength (the strength of the associations) as well as in the overall average reaches (the level of indirect connection of an individual). The females did not always show male avoidance behaviour, as evidenced by the thickness of the links between individuals in the sociograms, showing some of the bonds between males and females being as strong as the average of the population. However, there are cases of very weak bonds, with values smaller than 0.10 . The reduction of the values described above may be determined by the structure of the experimental tanks, in which stones and rocks offered small clefts where shark group of no more than two units could repair. It can be supposed that females, due to the shape of the shelters, could not occupy the same mass shelter and hence spread among the various cavities of the rocks. This suggests that females exhibit certain plasticity in their behaviour in order to reduce their contact with males. The tanks were set up with local sand and stones in order to recreate environmental conditions as similar as possible to natural ones, though this latter are anyway more dynamic and variable than those of the laboratory. This could be key information for interpreting the different results obtained with respect to Jacoby [26] on the Atlantic lesser spotted dogfish. In that case no shelter was available in the tanks, and the Index average real association of the female population showed an increase of the Index after the introduction of males. The presence of males seems to have a greater effect on individuals heaving weak links. Key individuals generally decreased their values of the Network analysis measures but in fact they were well linked within the population as shown by the sociograms. Peripheral individuals instead showed a greater variability with regard to the measures of the Network analysis, after the insertion of the males, with a tendency to increase these values. Still, in sociograms a wide variation is visible among individual, which changed their social position from one test phase to another. We can speculate that individuals initially not associated looked for more company to cope with the presence of males, even though these were also the individuals having more contacts with the males, as confirmed by comparing the Reach between analyses including or not data on the males. In the trial populations peripheral individuals had a much higher level of indirect connection when the male data were included in the analyses, indicating the presence of ties with the opposite sex, which in some cases stronger than the average. Actually, these bonds that males have with peripheral females and to a lesser degree also with better connected females prevent to seen an aggregation behavior of the females to avoid contact with the males. So we cannot confirm that in the Mediterranean lesser spotted dogfish the male avoidance behaviour of females by an aggregation mechanism is so marked as it was demonstrate in the Atlantic lesser spotted dogfish. In sociograms, it is visible that males established contact with all or almost all the female of the population, with a variable strength of the bonds. From studies carried out by D'Onghia [17] in the Aegean Sea and Massuti and Moranta [34] off the Balearic Islands coast, sexual segregation in adults of lesser spotted dogfish is often not found but, when present, it is linked to the body length. These studies may suggest that in the Mediterranean the two sexes have separate home-ranges, so that the presence of males does not involve an aggregation mechanism among the females to defend themselves from the presence of male coercion mechanisms. During the periods of this study no mating attempts or pursuits were registered by the males, though it should be noted that the observations were made during the day, in the resting phase and with lower activity of this species; this does not exclude that such events are likely to happen at night, when 
the animals are most active. Only a few bites were observed among females mostly during meal time observations carried out in the evenings. However even though that males have little effect on the female population, we must always keep in mind that their introduction alters the social structure of the females, responding with a lower average association index. This slackening of the bonds, shown by the reduction of the average real association Index, promotes an increased in the mixing and a more frequent exchange of preferred partners. The analysis of temporal stability in the T3 and T4 populations showed that associations already decayed over time becoming random and less lasting towards the end of the period of social definition, and the Association Lagged Rates were mostly close to the Null Association Rate. These results may indicate that males penetrate better in the social network in homogeneous populations where links are more uniform. Very interesting are the results of the analysis of temporal stability during the final recovery period, following the males' removal in which the trends of the Lagged Association Rates remained descending. This may indicate that the presence of males could have a lasting effect on the associations, in which the stability of bonds tends to be reduced over time even if $\mathrm{T} 4$ associations remain stable for the whole duration of the observation period.

\section{Conclusion}

The introduction of the male couples had a direct impact on the female social behaviour, causing a reduction of the aggregation behavior, visible in the reduction of the values of Strength, Reach and real average association Index. Moreover, the presence of males had stronger effects on the peripheral females with weaker bonds, which increased their connection within the social structure but, at the same time, had more contacts with the males. Furthermore, looking at the temporal analysis, the insertion of the males caused a reduction of the temporal stability of the relationships compared to the initial phase of social definition. These results, together with the very few literature about Mediterranean lesser spotted dogfish behaviour, may suggest that there is no aggregation behaviour or avoidance of males by females, as contacts and associations between sexes are frequent, perhaps indicating the absence of a real sexual segregation in the Mediterranean lesser spotted dogfish. This would be in accordance with observations on the spatial distribution of the two sexes made by D'Onghia [17] and Massuti and Moranta [34], indicating a reduced sexual segregation for this species in the Mediterranean. Finally, we cannot confirm the hypothesis that sexual coercion of Scyliorhinus canicula drives the strategy of avoidance by females causing an increase in aggregation in small marine refuges [6,7]. The results of this study suggest that there may be several factors may influence the formation of social networks, such as kinship, the different social roles of individuals and the parameters of the "small-world" networks. It would be worthwhile to examine these factors in order to increase our knowledge on sexual segregation and social behaviour in this species, and for sure this study will be followed by other experiments where shelters will not (or differently) be used, and where the sex-ratio test will be further altered. Our results suggest that the presence of males has a destructive effect on the social interactions of females. Therefore, it might be important to consider the sex ratio when animal are kept in captivity, adding an adequate number of shelters for such mechanism if behaviour of avoidance occurs in the future. This research on male-female relations and on social functions, regulating the sexual segregation of lesser spotted dogfish populations may have large implications and consequences on the management of these marine predators. Sharks are particularly vulnerable to exploitation due to a slow life history, a low reproductive rate and currently they undergo an excessive exploitation from fishing, often uncontrolled [35]. Aggregations of females in specific habitats may expose mature females, including pregnant ones, to a high risk of capture by the trawlers. Therefore, it is important to determine the factors that influence sexual segregation or sharing of home- ranges in elasmobranchs species to manage and conserve them as sexual differences could affect their spatial and temporal distribution leading to sex differences in their exploitation [36].

\section{References}

1. Sumpter JP, Dodd JM (1979) The annual reproductive cycle of the female lesser spotted dogfish, Scyliorhinus canicula $L$., and its endocrinal control. Journal of Fish Biology 15(6): 687-695.

2. Dodd JM (1983) Reproduction in cartlaginous fishes (Chondrichthyes). Reproduction- Endocrine Tissues and Hormones 9A: 31-95.

3. Rodriguez-Cabello C, Sanchez F, Olaso I (2007) Distribution patterns and sexual segregations of Scyliorhinus canicula (L.) in the Cantabrian Sea. Journal of Fish Biology 70(5): 1568-1586.

4. Ellis JR, Shackley SE (1997) The reproductive biology of Scyliorhinus canicula in the Bristol Channel, U.K. Journal of Fish Biology 51(2): 361-372.

5. Rodriguez-Cabello C, De La Gandara F, Sanchez F (1998) Preliminary results on growth and movements of dogfish Scyliorhinus canicula (Linnaeus, 1958) in the Cantabrian Sea. Oceanologica Acta 21: 363-370.

6. Sims DW, Nash JP, Morritt D (2001) Movements and activity of male and female dogfish in a tidal sea lough: alternative behavioral strategies and apparent sexual 
segregation. Marine Biology 139(6): 1165-1175.

7. Sims DW, Wearmouth VJ, Southall EJ, Hill JM, Moore P, et al. (2006) Hunt warm, rest cool: bioenergetic strategy underlying diel vertical migration of a benthic shark. Journal of Animal Ecology 75(1): 176-190.

8. Sims DW, Southall EJ, Wearmouth VJ, Hutchinson N, Budd GC, et al. (2005) Refuging behaviour in the nursehound Scyliorhinus stellaris (Chondrichthyes: Elasmobranchii): preliminary evidence from acoustic telemetry. Journal of the Marine Biological Association of the UK 85(5): 11371140 .

9. Sims DW, Southall EJ, Richardson AJ, Reid PC, Metclafe JD (2003) Seasonal movements and behaviour of basking sharks from archival tagging: No evidence of winter hibernation. Marine Ecology Progress Series. 248: 187196.

10. Sims DW (2003) Tractable models fot testing theories about natural strategies: foraging behaviour and habitat selection of free-ranging sharks. Journal of Fish Biology 63(S1): 53-73.

11. Kime DE, Hews EA (1982) The effect of temperature on steroid-biosynthesis by testes of the dogfish, Scyliorhinus canicula. Comparative Biochemistry and Physiology Part B 71(4): 675-679.

12. Sims DW (2005) Differences in habitat selection and reproductive strategies of male and female sharks. Sexual Segregation in Vertebrates: Ecology of the Two Sexes. Cambridge University Press, Cambridge.

13. Wey T, Blumstein DT, Shen W, Jorda'n F (2008) Social network analysis ofanimal behavior: a promising tool for the study of sociality. Animal Behaviour 75(2): 333-344.

14. Proulx SR, Promislow DEL, Phillips PC (2005) Network thinking in ecology and evolution. Trends Ecol Evol 20(6): 345-353.

15. Krause J, Butlin RK, Peuhkuri N, Pritchard VL (2000) The social organisation of fish shoals: a test of the predictive power of laboratory experiments for the field. Biol Rev 75(4): 477-501.

16. Croft DP, James R, Ward AJ, Botham MS, Mawdsley D, et al. (2005) Assortative interactions and social networks in fish. Oecologia 143(2): 211-219.

17. D’Onghia G, Matarrese A, Tursi A, Sion L (1995) Observations on the depth distribution pattern of the small-spotted catshark in the North Aegean Sea. Journal of Fish Biology 47(3): 421-426.
18. Bozzano A, Murgia R, Vallerga S, Hirano J, Archer S (2001) The photoreceptor system in the retinae of two dogfishes, Scyliorhinus canicula and Galeus melastomus: possible relationship with depth distribution and predatory lifestyle. Journal of Fish Biology 59(5): 12581278.

19. Compagno L, Dando M, Fowler S (2005) Sharks of the world. Princeton (NJ). Princeton University Press.

20. Whitehead H (2009) SOCPROG programs: analysing animal social structures. Behavioral Ecology and Sociobiology 63(5): 765-778.

21. Ginsberg JR, Young TP (1992) Measuring association between individuals or group in behavioral studies. Animal Behaviour 44(2): 377-379.

22. Bejder L, Fletcher D, Brager S (1998) A method for testing association patterns of social animals. Animal Behaviour 56(3): 719-725.

23. Newman MEJ (2003) Mixing patterns in networks. Physical Review E 67: 026126.

24. Newman MEJ (2003) The structure and function of complex networks. SIAM Review 45(2): 167- 256.

25. Newman MEJ (2004) Analysis of weighted networks. Physical Review E 70: 056131.

26. Jacoby DMP, Busawon DS, Sims DW (2010) Sex and social networking: the influence of male presence on social structure of female shark groups. Behavioural Ecology 21: 808-818.

27. Wasserman S, Faust K (1994) Social Network Analysis: Methods and Applications. Cambridge University Press, New York.

28. Hinde RA (1976) Interactions, relationships and social structure. Man 11(1): 1-17.

29. Whitehead H (1995) Investigating structure and temporal scale in social organizations using identified individuals. Behavioral Ecology 6(2): 199-208.

30. Whitehead H (2008) Analyzing animal societies: Quantitative Methods for VertebrateSocial Analysis. The University of Chicago Press, Chicago.

31. Whitehead H (1997) Analysing animal social structure. Animal Behavior 53(5): 1053-1067.

32. Whitehead H (2007) Selection of models of lagged identification rates and lagged association rates using AIC e QAIC. Communications in Statistics-Simulation and 
Computation 36(6): 1233-1246.

33. Croft DP, Albanese B, Arrowsmith BJ, Botham M, Webster $M$, et al. (2003) Sex- biased movement in the guppy (Poecilia reticulata). Oecologia 137(1): 447-452.

34. Massuti E, Moranta J (2003) Demersal assemblages and depth distribution of elasmobranchs from the continental shelf and slope off the Balearic Islands (western Mediterranean). Journal of Marine Science. 60(4): 753-766.
35. Dulvy N, Baum JK, Clarke S, Compagno L, Domingo A, et al. (2008) You can swim but you can't hide: the global status and conservation of oceanic pelagic sharks and rays. Aquatic Conservation: Marine and Freshwater Ecosystems 18(5): 459-482.

36. Mucientes GR, Queiroz N, Sousa LL, Tarroso P, Sims DW (2009) Sexual segregation of pelagic sharks and the potential threat from fisheries. Biology Letters 5(2): 156-159. 